the tracheostomy tubes within the false passage. Introducers of differing colors allow the identification of right and left main bronchial positions even when introducers and endobronchial tubes impair visualization of the distal airways.

We believe the use of rigid bronchoscopy and Frova introducers enables the exchange of endotracheal tubes in a controlled fashion and that superior visualization afforded by this technique minimizes the risk of further iatrogenic injury and therefore successful conservative management.

\section{References}

1. Delaney A, Bagshaw SM, Nalos M. Percutaneous dilatational tracheostomy versus surgical tracheostomy in critically ill patients: a systematic review and meta-analysis. Crit Care. 2006; 10:R55.

2. Gabor S, Renner H, Pinter H, Sankin O, Naier A, Tomaselli F, et al. Indications for surgery in tracheobronchial ruptures. Eur J Cardiothorac Surg. 2001;20:399-404.

3. Meyer M. Iatrogenic tracheobronchial lesions-a report on 13 cases. Thorac Cardiovasc Surg. 2001;49:115-9.

4. Beiderlinden M, Adamzik M, Peters J. Conservative treatment of tracheal injuries. Anesth Analg. 2005;100:210-4.

5. Conti M, Pougeoise M, Wurtz A, Porte H, Fourrier F, Ramon P, et al. Management of postintubation tracheobronchial ruptures. Chest. 2006;130:412-8

\title{
Quadruple valve replacement for acute endocarditis
}

Joerg Seeburger, MD, Heiner Groesdonk, MD, Michael Andrew Borger, MD, PhD, Denis Merk, MD, Joerg Ender, MD, Volkmar Falk, MD, PhD, Friedrich Wilhelm Mohr, MD, PhD, and Nicolas Doll, MD, PhD, Leipzig, Germany

A 76-year-old man presented with symptoms of heart failure, fever, and fatigue. His medical history included chronic renal and factor XIII insufficiency, diverticulosis, and chronic obstructive pulmonary disease. Transesophageal echocardiography showed endocarditis of the aortic, tricuspid, and pulmonary valves with severe insufficiency (Figure 1), and moderate mitral insufficiency with suspected vegetations (Figure 2).

Cardiac catheterization was normal. Broad-spectrum antibiotic coverage was initiated with ampicillin, gentamycin, and imipenem. After 10 days of conservative therapy, the patient underwent surgery because of ongoing symptoms of heart failure.

A median sternotomy and standard cannulation were performed. Moderate hypothermia and antegrade blood cardioplegia were administered. Because of large vegetations $(>1$ $\mathrm{cm}$ ) on all 4 valves, no reconstructive surgery was possible. The mitral valve was replaced (31-mm Epic, St Jude Medical, Minneapolis, Minn), followed by the tricuspid valve (33-mm Epic), the pulmonary valve (27-mm Freestyle, Medtronic Inc, Minneapolis, Minn), and the aortic valve (23-mm Epic). Because of third-degree arteriovenous block, a pacemaker lead (Medtronic Kappa) was attached epimyocardially and tunneled under the costal margin. The intraoperative course was otherwise uneventful, and the patient was weaned from cardiopulmonary bypass with small doses of inotropes.

From the Heartcenter Leipzig, Leipzig University, Leipzig, Germany.

Received for publication Jan 16, 2008; revisions received Jan 16, 2008; accepted for publication Feb 7, 2008.

Address for reprints: Joerg Seeburger, MD, Heartcenter Leipzig-University, Struempelstrasse 3904289 Leipzig, Germany (E-mail: j.seeburger@web.de).

J Thorac Cardiovasc Surg 2009; 137:1564-5

$0022-5223 / \$ 36.00$

Copyright (c) 2009 by The American Association for Thoracic Surgery doi:10.1016/j.jtcvs.2008.02.078
Postoperative bleeding required blood transfusion, coagulation factors, and recombinant activated factor VII.

Blood and aortic valve cultures revealed Enterococcus faecalis and Gemella morbillorum. Postoperative treatment was prolonged because of intracerebral bleeding with resultant left-sided hemiplegia, persistent upper gastrointestinal bleeding resulting in a Billroth II operation, and hemodialysis. Predischarge echocardiography showed excellent function of all 4 prosthetic valves. At 3 months follow-up, the patient is in rehabilitation and generally good health with gradually resolving neurologic symptoms.

\section{COMMENT}

Heart valve replacement for endocarditis is a relatively common operation, but the current case is notable for 2 reasons: (1) Endocarditis was caused by simultaneous infection

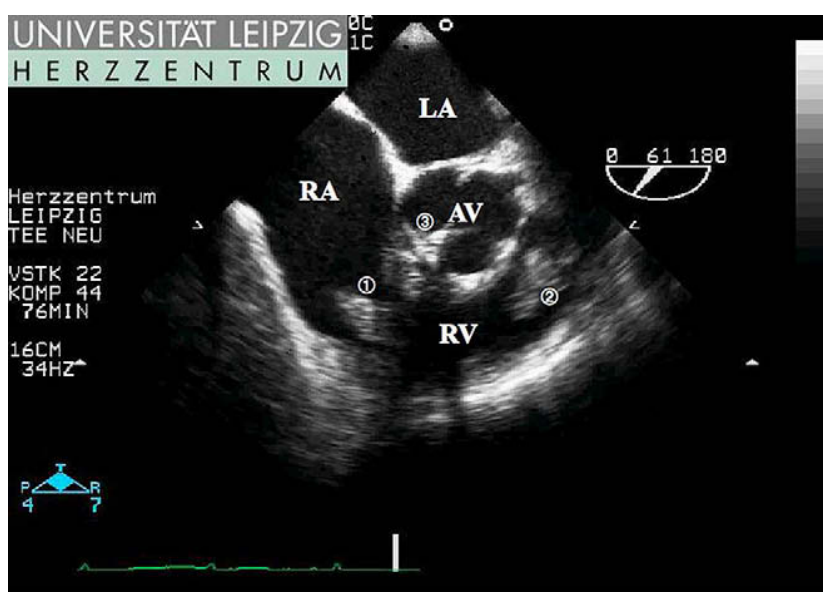

FIGURE 1. Transesophageal echocardiography showing severe endocarditis of the tricuspid (1), pulmonary (2), and aortic (3) valves. $L A$, Left atrium; $R A$, right atrium; $A V$, aortic valve; $R V$, right ventricle. 


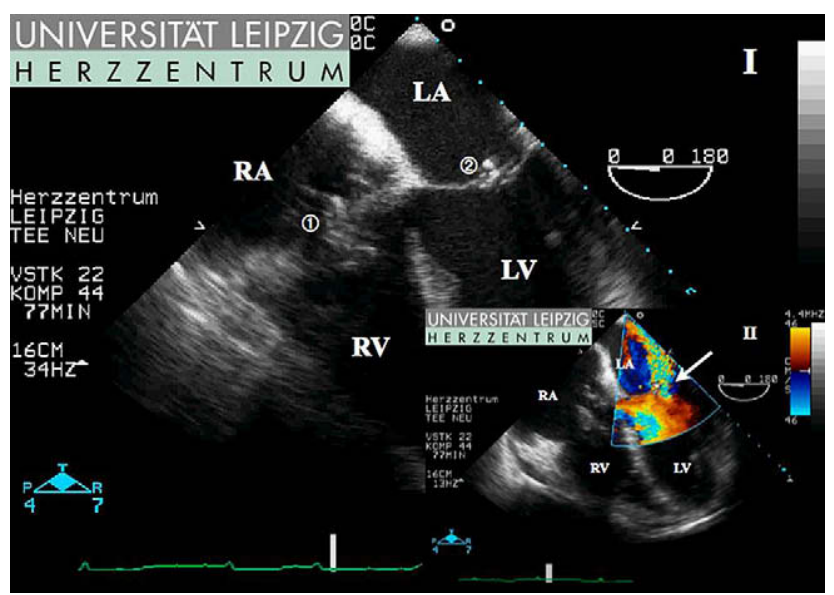

FIGURE 2. Severe endocarditis of the tricuspid valve ( 1 ) and suspected endocarditis of the mitral valve with moderate insufficiency (2). $L A$, Left atrium; $R A$, right atrium; $L V$, left ventricle; $R V$, right ventricle.

with E. faecalis and G. morbillorum, and (2) all cardiac valves were replaced in 1 operation. To the best of our knowledge, the current report is the first to describe successful quadruple valve replacement for acute endocarditis.

E. faecalis is known to cause endocarditis, as is G. morbillorum, but a simultaneous infection with both organisms has been described only once. ${ }^{1}$ Infection with E. faecalis can be extensive, as reported by Krake and colleagues ${ }^{2}$ in a patient with involvement of all cardiac valves. The patient in this case report did not undergo surgical treatment and subsequently died of sepsis. ${ }^{2}$

Involvement of all 4 heart valves can be observed with in other pathologic processes. Chiappini and colleagues ${ }^{3}$ reported on a patient with severe dysfunction of all valves secondary to carcinoid spread from the ileum. Their patient underwent valve replacement (tricuspid, pulmonary) and valve repair (aortic, mitral) with a good recovery. Hossack and colleagues ${ }^{4}$ also reported on a patient with rheumatic involvement of all valves treated with repeated operations, with a good long-term outcome. These 2 cases demonstrate that quadruple valve surgery can be successfully performed. Although the addition of pulmonary valve replacement adds to the complexity of the operation, previous investigators have demonstrated good long-term results with triple valve surgery. ${ }^{5}$

Our patient made a good recovery from a large and complex operation, despite several severe comorbidities before surgery and his complicated postoperative course. We think his recovery justifies such aggressive surgical therapy in selected patients with quadruple valve disease.

\section{CONCLUSIONS}

Replacement of all 4 heart valves in 1 operation is feasible in selected patients. Even in patients with endocarditis, a satisfactory outcome can be achieved.

\section{References}

1. Abboud R, Friart A. Two cases of isolated tricuspid endocarditis following colonic intervention. Acta Clin Belg. 1995;50:242-5

2. Krake PR, Zaman F, Tandon N. Native quadruple-valve endocarditis caused by Enterococcus faecalis. Tex Heart Inst J. 2004;31:90-2.

3. Chiappini B, Noirhomme P, Verhelst R, El Khoury G. Quadruple valve involvement in a patient with severe carcinoid heart disease. J Card Surg. 2006;21: 599-600.

4. Hossack KF, Hopeman AR, Sutherland EW 3rd. Long-term follow-up of a patien with quadruple valve replacement. Tex Heart Inst J. 1987;14:315-7.

5. Alsoufi B, Rao V, Borger MA, Maganti M, Armstrong S, Feindel CM, et al. Shortand long-term results of triple valve surgery in the modern era. Ann Thorac Surg. 2006;81:2172-8.

\title{
A new miniaturized cardiopulmonary bypass system reduces transfusion requirements during neonatal cardiac surgery: Initial experience in 13 consecutive patients
}

\author{
Andreas Koster, MD, ${ }^{\mathrm{a}}$ Michael Huebler, MD, ${ }^{\mathrm{b}}$ Wolfgang Boettcher, ECCP, ${ }^{\mathrm{c}}$ Mathias Redlin, MD, ${ }^{\mathrm{a}}$ Felix Berger, MD, ${ }^{\mathrm{d}}$ and \\ Roland Hetzer, MD, ${ }^{\mathrm{b}}$ Berlin, Germany
}

The potential detrimental effects of the transfusion of autologous blood products on patients' outcome is increasingly appreciated. ${ }^{1,2}$ This has promoted strategies to reduce the

\footnotetext{
From the Department of Anesthesia ${ }^{a}$ Department of Cardiovascular and Thoracic Surgery, ${ }^{\mathrm{b}}$ Department of Perfusion, ${ }^{\mathrm{c}}$ and Department of Cogenital Heart Disease, ${ }^{\mathrm{d}}$ Deutsches Herzzentrum Berlin, Berlin, Germany.

The study was supported by the "Deutsches Herzzentrum Berlin.",

Received for publication Jan 8, 2008; revisions received Feb 20, 2008; accepted for publication March 18, 2008.
}

consumption of donor blood products, as well as investigations assessing lower "critical" hemoglobin values as a trigger for transfusions. ${ }^{3}$ However, particularly in small infants

\footnotetext{
Address for reprints: Andreas Koster, MD, Department of Anesthesia, Deutsches Herzzentrum Berlin, Augustenburger Platz 1, D-13353 Berlin, Germany (E-mail: koster@dhzb.de).

J Thorac Cardiovasc Surg 2009; 137:1565-8

$0022-5223 / \$ 36.00$

Copyright (c) 2009 by The American Association for Thoracic Surgery doi:10.1016/j.jtcvs.2008.03.056
} 